\title{
An Iterative Method for Generalized Equilibrium Problems, Fixed Point Problems and Variational Inequality Problems
}

\author{
Qing-you Liu, ${ }^{1}$ Wei-you Zeng, ${ }^{2}$ and Nan-jing Huang ${ }^{2}$ \\ ${ }^{1}$ State Key Laboratory of Oil and Gas Reservoir Geology and Exploitation, Southwest Petroleum University, \\ Chengdu, Sichuan 610500, China \\ ${ }^{2}$ Department of Mathematics, Sichuan University, Chengdu, Sichuan 610064, China
}

Correspondence should be addressed to Nan-jing Huang, nanjinghuang@hotmail.com

Received 11 January 2009; Accepted 28 May 2009

Recommended by Fabio Zanolin

We introduce an iterative scheme by the viscosity approximation method for finding a common element of the set of solutions of generalized equilibrium problems, the set of common fixed points of infinitely many nonexpansive mappings, and the set of solutions of the variational inequality for $\alpha$-inverse-strongly monotone mappings in Hilbert spaces. We give some strong-convergence theorems under mild assumptions on parameters. The results presented in this paper improve and generalize the main result of Yao et al. (2007).

Copyright (C) 2009 Qing-you Liu et al. This is an open access article distributed under the Creative Commons Attribution License, which permits unrestricted use, distribution, and reproduction in any medium, provided the original work is properly cited.

\section{Introduction}

Let $C$ be a nonempty closed convex subset of a real Hilbert space $H$ and let $\Phi: C \times C \rightarrow \mathbb{R}$ be a bifunction, where $\mathbb{R}$ is the set of real numbers. Let $\Psi: C \rightarrow H$ be a nonlinear mapping. The generalized equilibrium problem (GEP) for $\Phi: C \times C \rightarrow \mathbb{R}$ and $\Psi: C \rightarrow H$ is to find $u \in C$ such that

$$
\Phi(u, v)+\langle\Psi u, v-u\rangle \geq 0 \quad \forall v \in C
$$

The set of solutions for the problem (1.1) is denoted by $\Omega$, that is,

$$
\Omega=\{u \in C: \Phi(u, v)+\langle\Psi u, v-u\rangle \geq 0, \forall v \in C\} .
$$


If $\Psi=0$ in (1.1), then GEP(1.1) reduces to the classical equilibrium problem (EP) and $\Omega$ is denoted by $\operatorname{EP}(\Phi)$, that is,

$$
\mathrm{EP}(\Phi)=\{u \in C: \Phi(u, v) \geq 0, \forall v \in C\}
$$

If $\Phi=0$ in (1.1), then GEP(1.1) reduces to the classical variational inequality and $\Omega$ is denoted by $\operatorname{VI}(\Psi, C)$, that is,

$$
\mathrm{VI}(\Psi, C)=\left\{u^{*} \in C:\left\langle\Psi u^{*}, v-u^{*}\right\rangle \geq 0, \forall v \in C\right\} .
$$

It is well known that GEP(1.1) contains as special cases, for instance, optimization problems, Nash equilibrium problems, complementarity problems, fixed point problems, and variational inequalities (see, e.g., [1-6] and the reference therein).

A mapping $A: C \rightarrow H$ is called $\alpha$-inverse-strongly monotone [7], if there exists a positive real number $\alpha$ such that

$$
\langle A x-A y, x-y\rangle \geq \alpha\|A x-A y\|^{2}
$$

for all $x, y \in C$. It is obvious that any $\alpha$-inverse-strongly monotone mapping $A$ is monotone and Lipschitz continuous. A mapping $S: C \rightarrow C$ is called nonexpansive if

$$
\|S x-S y\| \leq\|x-y\|
$$

for all $x, y \in C$. We denote by $F(S)$ the set of fixed points of $S$, that is, $F(S)=\{x \in C: x=$ $S(x)\}$. If $C \subset H$ is bounded, closed and convex and $S$ is a nonexpansive mappings of $C$ into itself, then $F(S)$ is nonempty (see [8]).

In 1997, Flåm and Antipin [9] introduced an iterative scheme of finding the best approximation to initial data when $\mathrm{EP}(\Phi)$ is nonempty and proved a strong convergence theorem. In 2003, Iusem and Sosa [10] presented some iterative algorithms for solving equilibrium problems in finite-dimensional spaces. They have also established the convergence of the algorithms. Recently, Huang et al. [11] studied the approximate method for solving the equilibrium problem and proved the strong convergence theorem for approximating the solutions of the equilibrium problem.

On the other hand, for finding an element of $F(S) \cap \operatorname{VI}(A, C)$, Takahashi and Toyoda [12] introduced the following iterative scheme:

$$
x_{n+1}=\alpha_{n} x_{n}+\left(1-\alpha_{n}\right) S P_{C}\left(x_{n}-\lambda_{n} A x_{n}\right), \quad n=0,1,2, \ldots,
$$

where $x_{0} \in C, P_{C}$ is metric projection of $H$ onto $C,\left\{\alpha_{n}\right\}$ is a sequence in $(0,1)$ and $\left\{\lambda_{n}\right\}$ is a sequence in $(0,2 \alpha)$. Further, Iiduka and Takahashi [13] introduced the following iterative scheme:

$$
x_{n+1}=\alpha_{n} u+\beta_{n} x_{n}+\gamma_{n} S P_{C}\left(x_{n}-\lambda_{n} A x_{n}\right),
$$

where $u, x_{0} \in C$, and proved the strong convergence theorems for iterative scheme (1.8) under some conditions on parameters. In 2007, S. Takahashi and W. Takahashi [14] introduced an 
iterative scheme by the viscosity approximation method for finding a common element of the set of an equilibrium problem and the set of fixed points of a nonexpansive mapping in Hilbert spaces. They also proved a strong convergence theorem which is connected with Combettes and Hirstoaga's result [3] and Wittmann's result [15]. Tada and W. Takahashi [16] introduced the Mann type iterative algorithm for finding a common element of the set of solutions of the $\mathrm{EP}(\Phi)$ and the set of common fixed points of nonexpansive mapping and obtained the weak convergence of the Mann type iterative algorithm. Yao et al. [17] introduced an iteration process for finding a common element of the set of solutions of the $\operatorname{EP}(\Phi)$ and the set of common fixed points of infinitely many nonexpansive mappings in Hilbert spaces. They proved a strong-convergence theorem under mild assumptions on parameters. Very recently, Moudafi [18] proposed an iterative algorithm for finding a common element of $\Omega \cap F(S)$, where $\Psi: C \rightarrow H$ is an $\alpha$-inverse-strongly monotone mapping, and obtained a weak convergence theorem. There are some related works, we refer to [19-22] and the references therein.

Inspired and motivated by the works mentioned above, in this paper, we introduce an iterative process for finding a common element of the set of common fixed points of infinitely many nonexpansive mappings, the set of solutions of GEP(1.1), and the solution set of the variational inequality problem for an $\alpha$-inverse-strongly monotone mapping in real Hilbert spaces. We give some strong-convergence theorems under mild assumptions on parameters. The results presented in this paper improve and generalize the main result of Yao et al. [17].

\section{Preliminaries}

Let $H$ be a real Hilbert space with inner product $\langle\cdot, \cdot\rangle$ and norm $\|\cdot\|$, and let $C$ be a closed convex subset of $H$. Then, for any $x \in H$, there exists a unique nearest point in $C$, denoted by $P_{C}(x)$, such that

$$
\left\|x-P_{C}(x)\right\| \leq\|x-y\| \quad \forall y \in C
$$

$P_{C}$ is called the metric projection of $H$ onto $C$. It is well known that $P_{C}$ is a nonexpansive mapping and satisfies

$$
\left\langle P_{C} x-P_{C} y, x-y\right\rangle \geq\left\|P_{C} x-P_{C} y\right\|^{2}
$$

for all $x, y \in H$. Furthermore, $P_{C}(x) \in C$ is characterized by the following properties:

$$
\begin{aligned}
\left\langle x-P_{C} x, y-P_{C} x\right\rangle & \leq 0, \\
\left\|x-P_{C} x\right\|^{2}+\left\|y-P_{C} x\right\|^{2} & \leq\|x-y\|^{2}
\end{aligned}
$$

for all $x \in H$ and $y \in C$. It is easy to see that

$$
u \in \operatorname{VI}(A, C) \Longleftrightarrow u=P_{C}(u-\lambda A u),
$$

where $\lambda>0$ is a parameter in $\mathbb{R}$. 
A set-valued mapping $T: H \rightarrow 2^{H}$ is called monotone if for all $x, y \in H, p \in T(x)$ and $q \in T(y)$ imply $\langle x-y, p-q\rangle \geq 0$. A monotone mapping $T: H \rightarrow 2^{H}$ is maximal if the graph $G(T)$ of $T$ is not properly contained in the graph of any other monotone mappings. It is known that a monotone mapping $T$ is maximal if and only if for $(x, p) \in H \times H,\langle x-y, p-q\rangle \geq 0$ for all $(y, q) \in G(T)$ implies $p \in T(x)$. Let $A: C \rightarrow H$ be a monotone, $L$-Lipschitz continuous mappings and let $N_{C} u$ be the normal cone to $C$ at $u \in C$, that is, $N_{C} u=\{w \in H:\langle u-v, w\rangle \geq$ $0, \forall v \in C\}$. Define

$$
T u= \begin{cases}A u+N_{C} u, & u \in C, \\ \emptyset, & u \notin C .\end{cases}
$$

Then $T$ is the maximal monotone and $0 \in T u$ if and only if $u \in \operatorname{VI}(A, C)$; see [23].

Let $\left\{T_{n}\right\}_{n=1}^{\infty}$ be a sequence of nonexpansive mappings of $C$ into itself and let $\left\{\pi_{n}\right\}_{n=1}^{\infty}$ be a sequence of nonnegative numbers in $[0,1]$. For any $n \geq 1$, define a mapping $S_{n}$ of $C$ into itself as follows:

$$
\begin{aligned}
U_{n, n+1} & =I, \\
U_{n, n} & =\pi_{n} T_{n} U_{n, n+1}+\left(1-\pi_{n}\right) I, \\
U_{n, n-1} & =\pi_{n-1} T_{n-1} U_{n, n}+\left(1-\pi_{n-1}\right) I, \\
\vdots & \\
U_{n, k} & =\pi_{k} T_{k} U_{n, k+1}+\left(1-\pi_{k}\right) I, \\
U_{n, k-1} & =\pi_{k-1} T_{k-1} U_{n, k}+\left(1-\pi_{k-1}\right) I, \\
\vdots & \\
U_{n, 2}= & \pi_{2} T_{2} U_{n, 3}+\left(1-\pi_{2}\right) I, \\
S_{n}= & U_{n, 1}=\pi_{1} T_{1} U_{n, 2}+\left(1-\pi_{1}\right) I .
\end{aligned}
$$

Such a mapping $S_{n}$ is called the $S$-mapping generated by $T_{n}, T_{n-1}, \ldots, T_{1}$ and $\pi_{n}, \pi_{n-1}, \ldots, \pi_{1}$ see [24]. It is obvious that $S_{n}$ is nonexpansive and if $x=T_{n} x$ then $x=U_{n, k}=S_{n} x$.

Lemma 2.1 (see [24]). Let $C$ be a nonempty closed convex subset of a real Hilbert space $H$. Let $\left\{T_{n}\right\}_{n=1}^{\infty}$ be a sequence of nonexpansive mappings of $C$ into itself such that $\bigcap_{n=1}^{\infty} F\left(T_{n}\right) \neq \emptyset$ and let $\left\{\pi_{n}\right\}_{n=1}^{\infty}$ be a sequence in $(0, \sigma]$ for some $\sigma \in(0,1)$. Then, for every $x \in C$ and $k \in \mathbb{N}=\{1,2, \ldots\}$, the limit $\lim _{n \rightarrow \infty} U_{n, k} x$ exists.

Remark 2.2 (see [17]). It can be known from Lemma 2.1 that if $D$ is a nonempty bounded subset of $C$, then for $\epsilon>0$, there exists $n_{0} \geq 1$ such that for all $n>n_{0}$

$$
\sup _{x \in D}\left\|U_{n, k} x-U_{k} x\right\| \leq \epsilon
$$


Fixed Point Theory and Applications

Using Lemma 2.1, one can define a mapping $S$ of $C$ into itself as follows:

$$
S x=\lim _{n \rightarrow \infty} S_{n} x=\lim _{n \rightarrow \infty} U_{n, 1} x
$$

for every $x \in C$. Such a mapping $S$ is called the $S$-mapping generated by $T_{1}, T_{2}, \ldots$ and $\pi_{1}, \pi_{2}, \ldots$ Since $S_{n}$ is nonexpansive, $S: C \rightarrow C$ is also nonexpansive. If $\left\{x_{n}\right\}$ is a bounded sequence in $C$, then we put $D=\left\{x_{n}: n \geq 0\right\}$. Hence, it is clear from Remark 2.2 that for an arbitrary $\epsilon>0$ there exists $N_{0} \in \mathbb{N}$ such that for all $n>N_{0}$

$$
\left\|S_{n} x_{n}-S x_{n}\right\|=\left\|U_{n, 1} x_{n}-U_{1} x_{n}\right\| \leq \sup _{x \in D}\left\|U_{n, 1} x-U_{1} x\right\| \leq \epsilon .
$$

This implies that

$$
\lim _{n \rightarrow \infty}\left\|S_{n} x_{n}-S x_{n}\right\|=0
$$

Since $T_{i}$ and $U_{n, i}$ are nonexpansive, we deduce that, for each $n \geq 1$,

$$
\begin{aligned}
\left\|S_{n+1} x_{n}-S_{n} x_{n}\right\| & =\left\|\pi_{1} T_{1} U_{n+1,2} x_{n}-\pi_{1} T_{1} U_{n, 2} x_{n}\right\| \\
& \leq \pi_{1}\left\|U_{n+1,2} x_{n}-U_{n, 2} x_{n}\right\| \\
& =\pi_{1}\left\|\pi_{2} T_{2} U_{n+1,3} x_{n}-\pi_{2} T_{2} U_{n, 3} x_{n}\right\| \\
& \leq \pi_{1} \pi_{2}\left\|U_{n+1,3} x_{n}-U_{n, 3} x_{n}\right\| \\
& \vdots \\
& \leq \prod_{i=1}^{n} \pi_{i}\left\|U_{n+1, n+1} x_{n}-U_{n, n+1} x_{n}\right\| \\
& \leq M \prod_{i=1}^{n} \pi_{i}
\end{aligned}
$$

for some constant $M \geq 0$.

Lemma 2.3 (see [24]). Let $C$ be a nonempty closed convex subset of a real Hilbert space $H$. Let $\left\{T_{n}\right\}_{n=1}^{\infty}$ be a sequence of nonexpansive mappings of $C$ into itself such that $\bigcap_{n=1}^{\infty} F\left(T_{n}\right) \neq \emptyset$, and let $\left\{\pi_{n}\right\}_{n=1}^{\infty}$ be a sequence in $(0, \sigma]$ for some $\sigma \in(0,1)$. Then, $F(S)=\bigcap_{n=1}^{\infty} F\left(T_{n}\right)$.

For solving the generalized equilibrium problem, we assume that the bifunction $\Phi$ : $C \times C \rightarrow \mathbb{R}$ satisfies the following conditions:

(a1) $\Phi(u, u)=0$ for all $u \in C$;

(a2) $\Phi$ is monotone, that is, $\Phi(u, v)+\Phi(v, u) \leq 0$ for all $u, v \in C$;

(a3) for each $u, v, w \in C, \lim _{t \downarrow 0} \Phi(t w+(1-t) u, v) \leq \Phi(u, v)$;

(a4) for each $u \in C, v \mapsto \Phi(u, v)$ is convex and lower semicontinuous. 
The following lemma appears implicitly in [1].

Lemma 2.4 (see [1]). Let $C$ be a nonempty closed convex subset of $H$, and let $\Phi$ be a bifunction from $C \times C$ into $\mathbb{R}$ satisfying (a1)-(a4). Let $r>0$ and $x \in H$. Then, there exists $u \in C$ such that

$$
\Phi(u, v)+\frac{1}{r}\langle v-u, u-x\rangle \geq 0 \quad \forall v \in C .
$$

The following lemma was also given in [3].

Lemma 2.5 (see [3]). Assume that $\Phi: C \times C \rightarrow \mathbb{R}$ satisfies (a1)-(a4). For $r>0$, define a mapping $T_{r}: H \rightarrow C$ as follows:

$$
T_{r}(x)=\left\{u \in C: \Phi(u, v)+\frac{1}{r}\langle v-u, u-x\rangle \geq 0, \forall v \in C\right\}
$$

for all $x \in H$. Then, the following hold:

(b1) $T_{r}$ is single-valued;

(b2) $T_{r}$ is firmly nonexpansive, that is, for any $x, y \in H,\left\|T_{r} x-T_{r} y\right\|^{2} \leq\left\langle T_{r} x-T_{r} y, x-y\right\rangle$;

(b3) $F\left(T_{r}\right)=E P(\Phi)$;

(b4) $E P(\Phi)$ is closed and convex.

Remark 2.6. Replacing $x$ with $x-r \Psi x \in H$ in (2.12), then there exists $u \in C$ such that

$$
\Phi(u, v)+\langle\Psi x, v-u\rangle+\frac{1}{r}\langle v-u, u-x\rangle \geq 0 \quad \forall v \in C .
$$

The following lemmas will be useful for proving the convergence result of this paper.

Lemma 2.7 (see [25]). Let $\left\{x_{n}\right\}$ and $\left\{z_{n}\right\}$ be bounded sequences in Banach space $E$, and let $\left\{\beta_{n}\right\}$ be a sequence in $[0,1]$. Suppose

$$
x_{n+1}=\left(1-\beta_{n}\right) z_{n}+\beta_{n} x_{n}
$$

for all integers $n \geq 1$. If

$$
\begin{gathered}
0<\liminf _{n \rightarrow \infty} \beta_{n} \leq \limsup _{n \rightarrow \infty} \beta_{n}<1, \\
\limsup _{n \rightarrow \infty}\left(\left\|z_{n+1}-z_{n}\right\|-\left\|x_{n+1}-x_{n}\right\|\right) \leq 0,
\end{gathered}
$$

then $\lim _{n \rightarrow \infty}\left\|z_{n}-x_{n}\right\|=0$.

Lemma 2.8 (see [26]). Assume $\left\{a_{n}\right\}$ is a sequence of nonnegative real numbers such that

$$
a_{n+1} \leq\left(1-\alpha_{n}\right) a_{n}+\delta_{n}, \quad n \geq 1,
$$


where $\left\{\alpha_{n}\right\}$ is a sequence in $(0,1)$ and $\left\{\delta_{n}\right\}$ is a sequence in $\mathbb{R}$ such that

(1) $\sum_{n=1}^{\infty} \alpha_{n}=\infty$;

(2) $\lim \sup _{n \rightarrow \infty}\left(\delta_{n} / \alpha_{n}\right) \leq 0$ or $\sum_{n=1}^{\infty}\left|\delta_{n}\right|<\infty$.

Then $\lim _{n \rightarrow \infty} a_{n}=0$.

\section{Main Results}

In this section, we deal with an iterative scheme by the approximation method for finding a common element of the set of common fixed points of infinitely many nonexpansive mappings, the set of solutions of GEP(1.1), and the solution set of the variational inequality problem for an $\alpha$-inverse-strongly monotone mapping in real Hilbert spaces.

Theorem 3.1. Let $C$ be a nonempty closed convex subset of a real Hilbert space $H$. Let $\Phi$ be a bifunction from $C \times C$ into $\mathbb{R}$ satisfying (a1)-(a4), $\Psi: C \rightarrow H$ an inverse-strongly monotone mapping with constant $\phi>0, A: C \rightarrow H$ an inverse-strongly monotone mapping with constant $\varrho>0, f: C \rightarrow C$ a contraction mapping with constant $\alpha \in[0,1)$. Let $S_{n}: C \rightarrow C$ be a S-mapping generated by $T_{1}, T_{2}, \ldots$ and $\pi_{1}, \pi_{2}, \ldots$ and $\bigcap_{n=1}^{\infty} F\left(T_{n}\right) \cap \Omega \cap V I(A, C) \neq \emptyset$, where sequence $\left\{T_{n}\right\}$ is nonexpansive and $\left\{\pi_{n}\right\}$ is a sequence in $(0, \sigma]$ for some $\sigma \in(0,1)$. For $x_{1} \in C$, suppose that $\left\{x_{n}\right\}$, $\left\{y_{n}\right\}$, and $\left\{u_{n}\right\}$ are generated by

$$
\begin{gathered}
\Phi\left(u_{n}, v\right)+\left\langle\Psi x_{n}, v-u_{n}\right\rangle+\frac{1}{r_{n}}\left\langle v-u_{n}, u_{n}-x_{n}\right\rangle \geq 0, \quad \forall v \in C, \\
y_{n}=P_{C}\left(u_{n}-\lambda_{n} A u_{n}\right), \\
x_{n+1}=\alpha_{n} f\left(x_{n}\right)+\beta_{n} x_{n}+\gamma_{n} S_{n} y_{n}
\end{gathered}
$$

for all $n \in \mathbb{N}$, where $\left\{\alpha_{n}\right\},\left\{\beta_{n}\right\}$, and $\left\{\gamma_{n}\right\}$ are three sequences in $[0,1],\left\{\lambda_{n}\right\}$ is a sequence in $(0, b]$ for some $0<b<2 \rho$ and $\left\{r_{n}\right\} \subset(0, d]$ for some $0<d<2 \phi$ satisfying

(i) $\alpha_{n}+\beta_{n}+\gamma_{n}=1$;

(ii) $\lim _{n \rightarrow \infty} \alpha_{n}=0$ and $\sum_{n=1}^{\infty} \alpha_{n}=\infty$;

(iii) $0<\liminf _{n \rightarrow \infty} \beta_{n} \leq \limsup _{n \rightarrow \infty} \beta_{n}<1$;

(iv) $\liminf _{n \rightarrow \infty} \lambda_{n}>0$ and $\lim _{n \rightarrow \infty}\left|\lambda_{n+1}-\lambda_{n}\right|=0$;

(v) $\liminf _{n \rightarrow \infty} r_{n}>0$ and $\lim _{n \rightarrow \infty}\left|r_{n+1}-r_{n}\right|=0$.

Then $\left\{x_{n}\right\},\left\{y_{n}\right\}$, and $\left\{u_{n}\right\}$ converge strongly to the point $z_{0} \in \bigcap_{n=1}^{\infty} F\left(T_{n}\right) \cap \Omega \cap \operatorname{VI}(A, C)$, where $z_{0}=P_{\bigcap_{n=1}^{\infty} F\left(T_{n}\right) \cap \Omega \cap V I(A, C)} f\left(z_{0}\right)$.

Proof. For any $x, y \in C$ and $r \in(0,2 \phi)$, we have

$$
\begin{aligned}
\|(I-r \Psi) x-(I-r \Psi) y\|^{2} & =\|(x-y)-r(\Psi x-\Psi y)\|^{2} \\
& =\|x-y\|^{2}-2 r\langle x-y, \Psi x-\Psi y\rangle+r^{2}\|\Psi x-\Psi y\|^{2} \\
& \leq\|x-y\|^{2}+r(r-2 \phi)\|\Psi x-\Psi y\|^{2} \\
& \leq\|x-y\|^{2}
\end{aligned}
$$


which implies that $I-r \Psi$ is nonexpansive. Remark 2.6 implies that the sequences $\left\{u_{n}\right\}$ and $\left\{x_{n}\right\}$ are well defined. In view of the iterative sequence (3.1), we have

$$
\begin{aligned}
0 & \leq \Phi\left(u_{n}, v\right)+\left\langle\Psi x_{n}, v-u_{n}\right\rangle+\frac{1}{r_{n}}\left\langle v-u_{n}, u_{n}-x_{n}\right\rangle \\
& =\Phi\left(u_{n}, v\right)+\frac{1}{r_{n}}\left\langle v-u_{n}, u_{n}-\left(x_{n}-r_{n} \Psi x_{n}\right)\right\rangle .
\end{aligned}
$$

It follows from Lemma 2.5 that $u_{n}=T_{r_{n}}\left(x_{n}-r_{n} \Psi x_{n}\right)$ for all $n \geq 1$. Let $z^{*} \in \bigcap_{n=1}^{\infty} F\left(T_{n}\right) \cap \Omega \cap$ $\operatorname{VI}(A, C)$. For each $n \geq 1$, we have $z^{*}=S_{n}\left(z^{*}\right)=T_{r_{n}}\left(z^{*}-r_{n} \Psi z^{*}\right)$. By Lemma 2.5,

$$
\begin{aligned}
\left\|u_{n}-z^{*}\right\|^{2} & =\left\|T_{r_{n}}\left(x_{n}-r_{n} \Psi x_{n}\right)-T_{r_{n}}\left(z^{*}-r_{n} \Psi z^{*}\right)\right\|^{2} \\
\leq & \left\langle u_{n}-z^{*},\left(x_{n}-r_{n} \Psi x_{n}\right)-\left(z^{*}-r_{n} \Psi z^{*}\right)\right\rangle \\
= & \frac{1}{2}\left(\left\|u_{n}-z^{*}\right\|^{2}+\left\|\left(x_{n}-r_{n} \Psi x_{n}\right)-\left(z^{*}-r_{n} \Psi z^{*}\right)\right\|^{2}\right. \\
& \left.\quad-\left\|\left(u_{n}-z^{*}\right)-\left(\left(x_{n}-r_{n} \Psi x_{n}\right)-\left(z^{*}-r_{n} \Psi z^{*}\right)\right)\right\|^{2}\right)
\end{aligned}
$$

and so (3.2) implies that

$$
\begin{aligned}
\left\|u_{n}-z^{*}\right\|^{2} & \leq\left\|\left(x_{n}-r_{n} \Psi x_{n}\right)-\left(z^{*}-r_{n} \Psi z^{*}\right)\right\|^{2}-\left\|\left(u_{n}-x_{n}\right)-r_{n}\left(\Psi z^{*}-\Psi x_{n}\right)\right\|^{2} \\
& \leq\left\|x_{n}-z^{*}\right\|^{2}-\left\|\left(u_{n}-x_{n}\right)-r_{n}\left(\Psi z^{*}-\Psi x_{n}\right)\right\|^{2} \\
& \leq\left\|x_{n}-z^{*}\right\|^{2} .
\end{aligned}
$$

For $z^{*} \in \operatorname{VI}(A, C)$, we have $z^{*}=P_{C}\left(z^{*}-\lambda_{n} A z^{*}\right)$ from (2.4). Since $P_{C}$ is a nonexpansive mapping and $A$ is an inverse-strongly monotone mapping with constant $\rho>0$, by (3.1), we have

$$
\begin{aligned}
\left\|y_{n}-z^{*}\right\|^{2} & =\left\|P_{C}\left(u_{n}-\lambda_{n} A u_{n}\right)-P_{C}\left(z^{*}-\lambda_{n} A z^{*}\right)\right\|^{2} \\
& \leq\left\|\left(u_{n}-\lambda_{n} A u_{n}\right)-\left(z^{*}-\lambda_{n} A z^{*}\right)\right\|^{2} \\
& \leq\left\|u_{n}-z^{*}\right\|^{2}+\lambda_{n}\left(\lambda_{n}-2 \varrho\right)\left\|A u_{n}-A z^{*}\right\|^{2} \\
& \leq\left\|u_{n}-z^{*}\right\|^{2} .
\end{aligned}
$$

Thus, (3.5) and (3.6) imply that

$$
\left\|y_{n}-z^{*}\right\| \leq\left\|u_{n}-z^{*}\right\| \leq\left\|x_{n}-z^{*}\right\|,
$$


and so

$$
\begin{aligned}
\left\|x_{n+1}-z^{*}\right\| & =\left\|\alpha_{n} f\left(x_{n}\right)+\beta_{n} x_{n}+\gamma_{n} S_{n} y_{n}-z^{*}\right\| \\
& \leq \alpha_{n}\left\|f\left(x_{n}\right)-z^{*}\right\|+\beta_{n}\left\|x_{n}-z^{*}\right\|+\gamma_{n}\left\|S_{n} y_{n}-z^{*}\right\| \\
& \leq \alpha_{n}\left(\left\|f\left(x_{n}\right)-f\left(z^{*}\right)\right\|+\left\|f\left(z^{*}\right)-z^{*}\right\|\right)+\beta_{n}\left\|x_{n}-z^{*}\right\|+\gamma_{n}\left\|S_{n} y_{n}-S_{n} z^{*}\right\| \\
& \leq \alpha_{n}\left(\alpha\left\|x_{n}-z^{*}\right\|+\left\|f\left(z^{*}\right)-z^{*}\right\|\right)+\beta_{n}\left\|x_{n}-z^{*}\right\|+\gamma_{n}\left\|y_{n}-z^{*}\right\| \\
& \leq \alpha_{n}\left(\alpha\left\|x_{n}-z^{*}\right\|+\left\|f\left(z^{*}\right)-z^{*}\right\|\right)+\beta_{n}\left\|x_{n}-z^{*}\right\|+\gamma_{n}\left\|x_{n}-z^{*}\right\| \\
& =\left[1-\alpha_{n}(1-\alpha)\right]\left\|x_{n}-z^{*}\right\|+\alpha_{n}(1-\alpha) \frac{\left\|f\left(z^{*}\right)-z^{*}\right\|}{1-\alpha} \\
& \leq \max \left\{\left\|x_{1}-z^{*}\right\|, \frac{\left\|f\left(z^{*}\right)-z^{*}\right\|}{1-\alpha}\right\} .
\end{aligned}
$$

This implies that $\left\{x_{n}\right\}$ is bounded. Therefore, $\left\{u_{n}\right\},\left\{y_{n}\right\},\left\{\Psi x_{n}\right\},\left\{A u_{n}\right\}$, and $\left\{S_{n} y_{n}\right\}$ are also bounded.

From $u_{n}=T_{r_{n}}\left(x_{n}-r_{n} \Psi x_{n}\right)$ and $u_{n+1}=T_{r_{n+1}}\left(x_{n+1}-r_{n+1} \Psi x_{n+1}\right)$, we have

$$
\begin{gathered}
\Phi\left(u_{n}, v\right)+\left\langle\Psi x_{n}, v-u_{n}\right\rangle+\frac{1}{r_{n}}\left\langle v-u_{n}, u_{n}-x_{n}\right\rangle \geq 0 \quad \forall v \in C, \\
\Phi\left(u_{n+1}, v\right)+\left\langle\Psi x_{n+1}, v-u_{n+1}\right\rangle+\frac{1}{r_{n+1}}\left\langle v-u_{n+1}, u_{n+1}-x_{n+1}\right\rangle \geq 0 \quad \forall v \in C .
\end{gathered}
$$

Putting $v=u_{n+1}$ in (3.9) and $v=u_{n}$ in (3.10), we get

$$
\begin{gathered}
\Phi\left(u_{n}, u_{n+1}\right)+\left\langle\Psi x_{n}, u_{n+1}-u_{n}\right\rangle+\frac{1}{r_{n}}\left\langle u_{n+1}-u_{n}, u_{n}-x_{n}\right\rangle \geq 0, \\
\Phi\left(u_{n+1}, u_{n}\right)+\left\langle\Psi x_{n+1}, u_{n}-u_{n+1}\right\rangle+\frac{1}{r_{n+1}}\left\langle u_{n}-u_{n+1}, u_{n+1}-x_{n+1}\right\rangle \geq 0 .
\end{gathered}
$$

Adding the above two inequalities, the monotonicity of $\Phi$ implies that

$$
\left\langle\Psi x_{n+1}-\Psi x_{n}, u_{n}-u_{n+1}\right\rangle+\left\langle u_{n}-u_{n+1}, \frac{u_{n+1}-x_{n+1}}{r_{n+1}}-\frac{u_{n}-x_{n}}{r_{n}}\right\rangle \geq 0,
$$

and so

$$
\begin{aligned}
0 & \leq\left\langle u_{n}-u_{n+1}, r_{n}\left(\Psi x_{n+1}-\Psi x_{n}\right)+\frac{r_{n}}{r_{n+1}}\left(u_{n+1}-x_{n+1}\right)-\left(u_{n}-x_{n}\right)\right\rangle \\
& =\left\langle u_{n+1}-u_{n}, u_{n}-u_{n+1}+\left(1-\frac{r_{n}}{r_{n+1}}\right) u_{n+1}+\left(x_{n+1}-r_{n} \Psi x_{n+1}\right)-\left(x_{n}-r_{n} \Psi x_{n}\right)-x_{n+1}+\frac{r_{n}}{r_{n+1}} x_{n+1}\right\rangle \\
& =\left\langle u_{n+1}-u_{n}, u_{n}-u_{n+1}+\left(1-\frac{r_{n}}{r_{n+1}}\right)\left(u_{n+1}-x_{n+1}\right)+\left(x_{n+1}-r_{n} \Psi x_{n+1}\right)-\left(x_{n}-r_{n} \Psi x_{n}\right)\right\rangle .
\end{aligned}
$$


It follows from (3.2) that

$$
\left\|u_{n+1}-u_{n}\right\|^{2} \leq\left\|u_{n+1}-u_{n}\right\|\left\{\left|1-\frac{r_{n}}{r_{n+1}}\right|\left\|u_{n+1}-x_{n+1}\right\|+\left\|x_{n+1}-x_{n}\right\|\right\},
$$

and hence

$$
\left\|u_{n+1}-u_{n}\right\| \leq\left|1-\frac{r_{n}}{r_{n+1}}\right|\left\|u_{n+1}-x_{n+1}\right\|+\left\|x_{n+1}-x_{n}\right\| .
$$

From (3.1),

$$
\begin{aligned}
\left\|y_{n+1}-y_{n}\right\| & =\left\|P_{C}\left(u_{n+1}-\lambda_{n+1} A u_{n+1}\right)-P_{C}\left(u_{n}-\lambda_{n} A u_{n}\right)\right\| \\
& \leq\left\|\left(u_{n+1}-\lambda_{n+1} A u_{n+1}\right)-\left(u_{n}-\lambda_{n} A u_{n}\right)\right\| \\
& \leq\left\|\left(u_{n+1}-\lambda_{n+1} A u_{n+1}\right)-\left(u_{n}-\lambda_{n} A u_{n+1}\right)\right\|+\left|\lambda_{n+1}-\lambda_{n}\right|\left\|A u_{n}\right\| \\
& \leq\left\|u_{n+1}-u_{n}\right\|+\left|\lambda_{n+1}-\lambda_{n}\right|\left\|A u_{n}\right\| .
\end{aligned}
$$

Putting

$$
z_{n}=\frac{\alpha_{n}}{1-\beta_{n}} f\left(x_{n}\right)+\frac{\gamma_{n}}{1-\beta_{n}} S_{n} y_{n}
$$

we have

$$
x_{n+1}=\beta_{n} x_{n}+\left(1-\beta_{n}\right) z_{n} .
$$

Obviously, we get

$$
\begin{aligned}
\left\|z_{n+1}-z_{n}\right\|= & \left\|\frac{\alpha_{n+1}}{1-\beta_{n+1}} f\left(x_{n+1}\right)+\frac{\gamma_{n+1}}{1-\beta_{n+1}} S_{n+1} y_{n+1}-\left(\frac{\alpha_{n}}{1-\beta_{n}} f\left(x_{n}\right)+\frac{\gamma_{n}}{1-\beta_{n}} S_{n} y_{n}\right)\right\| \\
\leq & \frac{\alpha_{n+1}}{1-\beta_{n+1}}\left\|f\left(x_{n+1}\right)-f\left(x_{n}\right)\right\|+\left|\frac{\alpha_{n+1}}{1-\beta_{n+1}}-\frac{\alpha_{n}}{1-\beta_{n}}\right|\left\|f\left(x_{n}\right)\right\| \\
& +\frac{\gamma_{n+1}}{1-\beta_{n+1}}\left\|S_{n+1} y_{n+1}-S_{n} y_{n}\right\|+\left|\frac{\gamma_{n+1}}{1-\beta_{n+1}}-\frac{\gamma_{n}}{1-\beta_{n}}\right|\left\|S_{n} y_{n}\right\| \\
\leq & \frac{\alpha_{n+1}}{1-\beta_{n+1}} \alpha\left\|x_{n+1}-x_{n}\right\|+\left|\frac{\alpha_{n+1}}{1-\beta_{n+1}}-\frac{\alpha_{n}}{1-\beta_{n}}\right|\left\|f\left(x_{n}\right)\right\| \\
& +\left|\frac{\alpha_{n}}{1-\beta_{n}}-\frac{\alpha_{n+1}}{1-\beta_{n+1}}\right|\left\|S_{n} y_{n}\right\|+\left(1-\frac{\alpha_{n+1}}{1-\beta_{n+1}}\right)\left\|S_{n+1} y_{n+1}-S_{n} y_{n}\right\| .
\end{aligned}
$$


Fixed Point Theory and Applications

From (2.11) and (3.16), we have

$$
\begin{aligned}
\left\|S_{n+1} y_{n+1}-S_{n} y_{n}\right\| & \leq\left\|S_{n+1} y_{n+1}-S_{n+1} y_{n}\right\|+\left\|S_{n+1} y_{n}-S_{n} y_{n}\right\| \\
& \leq\left\|y_{n+1}-y_{n}\right\|+M \prod_{i=1}^{n} \pi_{i} \\
& \leq\left\|u_{n+1}-u_{n}\right\|+\left|\lambda_{n+1}-\lambda_{n}\right|\left\|A u_{n}\right\|+M \prod_{i=1}^{n} \pi_{i}
\end{aligned}
$$

for some constant $M \geq 0$. Combining (3.15), (3.19), and (3.20), we deduce

$$
\begin{aligned}
\| z_{n+1}- & z_{n}\|-\| x_{n+1}-x_{n} \| \\
\leq & \frac{\alpha_{n+1}(\alpha-1)}{1-\beta_{n+1}}\left\|x_{n+1}-x_{n}\right\|+\left|\frac{\alpha_{n+1}}{1-\beta_{n+1}}-\frac{\alpha_{n}}{1-\beta_{n}}\right|\left(\left\|f\left(x_{n}\right)\right\|+\left\|S_{n} y_{n}\right\|\right) \\
& \quad+\frac{\gamma_{n+1}}{1-\beta_{n+1}}\left(M \prod_{i=1}^{n} \pi_{i}+\left|\lambda_{n+1}-\lambda_{n}\right| \mid A u_{n}\left\|+\frac{\left|r_{n+1}-r_{n}\right|}{r_{n+1}}\right\| u_{n+1}-x_{n+1} \|\right) .
\end{aligned}
$$

It is easy to check that

$$
\lim _{n \rightarrow \infty}\left|\frac{\alpha_{n+1}}{1-\beta_{n+1}}-\frac{\alpha_{n}}{1-\beta_{n}}\right|=0, \quad \lim _{n \rightarrow \infty} \prod_{i=1}^{n} \pi_{i}=0, \quad \lim _{n \rightarrow \infty}\left|r_{n+1}-r_{n}\right|=0,
$$

and so

$$
\limsup _{n \rightarrow \infty}\left(\left\|z_{n+1}-z_{n}\right\|-\left\|x_{n+1}-x_{n}\right\|\right) \leq 0
$$

Thus, by Lemma 2.7, we obtain $\lim _{n \rightarrow \infty}\left\|z_{n}-x_{n}\right\|=0$. It then follows that

$$
\lim _{n \rightarrow \infty}\left\|x_{n+1}-x_{n}\right\|=\lim _{n \rightarrow \infty}\left(1-\beta_{n}\right)\left\|z_{n}-x_{n}\right\|=0 .
$$

By (3.15) and (3.16), we have

$$
\lim _{n \rightarrow \infty}\left\|y_{n+1}-y_{n}\right\|=\lim _{n \rightarrow \infty}\left\|u_{n+1}-u_{n}\right\|=0
$$


Since $x_{n+1}=\alpha_{n} f\left(x_{n}\right)+\beta_{n} x_{n}+\gamma_{n} S_{n} y_{n}$, we get

$$
\begin{aligned}
\left\|x_{n+1}-\frac{\beta_{n} x_{n}+\gamma_{n} S_{n} y_{n}}{1-\alpha_{n}}\right\| & =\left\|\alpha_{n} f\left(x_{n}\right)+\left(1-\frac{1}{1-\alpha_{n}}\right)\left(\beta_{n} x_{n}+\gamma_{n} S_{n} y_{n}\right)\right\| \\
& =\alpha_{n}\left\|f\left(x_{n}\right)-\frac{\beta_{n} x_{n}+\gamma_{n} S_{n} y_{n}}{1-\alpha_{n}}\right\| \longrightarrow 0 .
\end{aligned}
$$

On the other hand, for $\alpha_{n}+\beta_{n}+\gamma_{n}=1$,

$$
x_{n}-\frac{\beta_{n} x_{n}+\gamma_{n} S_{n} y_{n}}{1-\alpha_{n}}=\frac{\gamma_{n}}{1-\alpha_{n}}\left(x_{n}-S_{n} y_{n}\right)
$$

It follows that

$$
\begin{aligned}
\gamma_{n}\left\|x_{n}-S_{n} y_{n}\right\| & =\left(1-\alpha_{n}\right)\left\|x_{n}-\frac{\beta_{n} x_{n}+\gamma_{n} S_{n} y_{n}}{1-\alpha_{n}}\right\| \\
& \leq\left(1-\alpha_{n}\right)\left(\left\|x_{n}-x_{n+1}\right\|+\left\|x_{n+1}-\frac{\beta_{n} x_{n}+\gamma_{n} S_{n} y_{n}}{1-\alpha_{n}}\right\|\right) \rightarrow 0 .
\end{aligned}
$$

It is easy to see that $\liminf _{n \rightarrow \infty} \gamma_{n}>0$ and hence $\lim _{n \rightarrow \infty}\left\|x_{n}-S_{n} y_{n}\right\|=0$.

From (3.5) and (3.6), we obtain

$$
\begin{aligned}
\left\|x_{n+1}-z^{*}\right\|^{2} & \leq \alpha_{n}\left\|f\left(x_{n}\right)-z^{*}\right\|^{2}+\beta_{n}\left\|x_{n}-z^{*}\right\|^{2}+\gamma_{n}\left\|S_{n} y_{n}-z^{*}\right\|^{2} \\
& \leq \alpha_{n}\left\|f\left(x_{n}\right)-z^{*}\right\|^{2}+\beta_{n}\left\|x_{n}-z^{*}\right\|^{2}+\gamma_{n}\left\|y_{n}-z^{*}\right\|^{2} \\
& \leq \alpha_{n}\left\|f\left(x_{n}\right)-z^{*}\right\|^{2}+\beta_{n}\left\|x_{n}-z^{*}\right\|^{2}+\gamma_{n}\left\{\left\|u_{n}-z^{*}\right\|^{2}+\lambda_{n}\left(\lambda_{n}-2 \varphi\right)\left\|A u_{n}-A z^{*}\right\|^{2}\right\} \\
& \leq \alpha_{n}\left\|f\left(x_{n}\right)-z^{*}\right\|^{2}+\left\|x_{n}-z^{*}\right\|^{2}+\gamma_{n} \lambda_{n}\left(\lambda_{n}-2 \varrho\right)\left\|A u_{n}-A z^{*}\right\|^{2} .
\end{aligned}
$$

Since lim inf $r_{n}>0$, without loss of generality, we may assume that there exists a real number $a>0$ such that $r_{n}>a$ for all $n \in \mathbb{N}$. Therefore, we have

$$
\begin{aligned}
& \gamma_{n} a(2 \rho-b)\left\|A u_{n}-A z^{*}\right\|^{2} \\
& \quad \leq \alpha_{n}\left\|f\left(x_{n}\right)-z^{*}\right\|^{2}+\left\|x_{n}-z^{*}\right\|^{2}-\left\|x_{n+1}-z^{*}\right\|^{2} \\
& \quad \leq \alpha_{n}\left\|f\left(x_{n}\right)-z^{*}\right\|^{2}+\left\|x_{n}-x_{n+1}\right\|\left(\left\|x_{n}-z^{*}\right\|+\left\|x_{n+1}-z^{*}\right\|\right) .
\end{aligned}
$$


Since $\alpha_{n} \rightarrow 0,\left\|x_{n}-x_{n+1}\right\| \rightarrow 0$ and $\left\{x_{n}\right\}$ is bounded, (3.30) implies that $\left\|A u_{n}-A z^{*}\right\| \rightarrow 0$ as $n \rightarrow \infty$. From (2.2), we have

$$
\begin{aligned}
\left\|y_{n}-z^{*}\right\|^{2}= & \left\|P_{C}\left(u_{n}-\lambda_{n} A u_{n}\right)-P_{C}\left(z^{*}-\lambda_{n} A z^{*}\right)\right\|^{2} \\
\leq & \left\langle\left(u_{n}-\lambda_{n} A u_{n}\right)-\left(z^{*}-\lambda_{n} A z^{*}\right), y_{n}-z^{*}\right\rangle \\
= & \frac{1}{2}\left\{\left\|\left(u_{n}-\lambda_{n} A u_{n}\right)-\left(z^{*}-\lambda_{n} A z^{*}\right)\right\|^{2}+\left\|y_{n}-z^{*}\right\|^{2}\right. \\
& \left.\quad-\left\|\left(u_{n}-y_{n}\right)-\lambda_{n}\left(A u_{n}-A z^{*}\right)\right\|^{2}\right\} \\
\leq & \frac{1}{2}\left\{\left\|u_{n}-z^{*}\right\|^{2}+\left\|y_{n}-z^{*}\right\|^{2}\right. \\
& \left.\quad-\left(\left\|u_{n}-y_{n}\right\|^{2}-2 \lambda_{n}\left\langle u_{n}-y_{n}, A u_{n}-A z^{*}\right\rangle+\lambda_{n}^{2}\left\|A u_{n}-A z^{*}\right\|^{2}\right)\right\},
\end{aligned}
$$

and so

$$
\left\|y_{n}-z^{*}\right\|^{2} \leq\left\|u_{n}-z^{*}\right\|^{2}-\left\|u_{n}-y_{n}\right\|^{2}+2 \lambda_{n}\left\langle u_{n}-y_{n}, A u_{n}-A z^{*}\right\rangle-\lambda_{n}^{2}\left\|A u_{n}-A z^{*}\right\|^{2}
$$

It follows that

$$
\begin{aligned}
\left\|x_{n+1}-z^{*}\right\| \leq & \alpha_{n}\left\|f\left(x_{n}\right)-z^{*}\right\|^{2}+\beta_{n}\left\|x_{n}-z^{*}\right\|^{2}+\gamma_{n}\left\|y_{n}-z^{*}\right\|^{2} \\
\leq & \alpha_{n}\left\|f\left(x_{n}\right)-z^{*}\right\|^{2}+\beta_{n}\left\|x_{n}-z^{*}\right\|^{2} \\
& +\gamma_{n}\left(\left\|u_{n}-z^{*}\right\|^{2}-\left\|u_{n}-y_{n}\right\|^{2}+2 \lambda_{n}\left\langle u_{n}-y_{n}, A u_{n}-A z^{*}\right\rangle-\lambda_{n}^{2}\left\|A u_{n}-A z^{*}\right\|^{2}\right) \\
\leq & \alpha_{n}\left\|f\left(x_{n}\right)-z^{*}\right\|^{2}+\left\|x_{n}-z^{*}\right\|^{2}-\gamma_{n}\left\|u_{n}-y_{n}\right\|^{2} \\
& +2 \gamma_{n} \lambda_{n}\left\langle u_{n}-y_{n}, A u_{n}-A z^{*}\right\rangle-\gamma_{n} \lambda_{n}^{2}\left\|A u_{n}-A z^{*}\right\|^{2}
\end{aligned}
$$

which implies that

$$
\begin{aligned}
\gamma_{n}\left\|u_{n}-y_{n}\right\|^{2} \leq & \alpha_{n}\left\|f\left(x_{n}\right)-z^{*}\right\|^{2}+\left\|x_{n}-z^{*}\right\|^{2}-\left\|x_{n+1}-z^{*}\right\|^{2} \\
& +2 \gamma_{n} \lambda_{n}\left\langle u_{n}-y_{n}, A u_{n}-A z^{*}\right\rangle \\
\leq & \alpha_{n}\left\|f\left(x_{n}\right)-z^{*}\right\|^{2}+\left\|x_{n}-x_{n+1}\right\|\left(\left\|x_{n}-z^{*}\right\|+\left\|x_{n+1}-z^{*}\right\|\right) \\
& +2 \gamma_{n} \lambda_{n}\left\|A u_{n}-A z^{*}\right\|\left\|u_{n}-y_{n}\right\| .
\end{aligned}
$$


Since $\alpha_{n} \rightarrow 0,\left\|x_{n}-x_{n+1}\right\| \rightarrow 0,\left\|A u_{n}-A z^{*}\right\| \rightarrow 0$, and the sequences $\left\{x_{n}\right\},\left\{y_{n}\right\}$ and $\left\{u_{n}\right\}$ are bounded, it follows from (3.34) that $\lim _{n \rightarrow \infty}\left\|u_{n}-y_{n}\right\|=0$. On the other hand, from (3.5), we have

$$
\begin{aligned}
\left\|x_{n+1}-z^{*}\right\|^{2} \leq & \alpha_{n}\left\|f\left(x_{n}\right)-z^{*}\right\|^{2}+\beta_{n}\left\|x_{n}-z^{*}\right\|^{2}+\gamma_{n}\left\|y_{n}-z^{*}\right\|^{2} \\
\leq & \alpha_{n}\left\|f\left(x_{n}\right)-z^{*}\right\|^{2}+\beta_{n}\left\|x_{n}-z^{*}\right\|^{2}+\gamma_{n}\left\|u_{n}-z^{*}\right\|^{2} \\
\leq & \alpha_{n}\left\|f\left(x_{n}\right)-z^{*}\right\|^{2}+\beta_{n}\left\|x_{n}-z^{*}\right\|^{2} \\
& +\gamma_{n}\left(\left\|x_{n}-z^{*}\right\|^{2}+r_{n}\left(r_{n}-2 \phi\right)\left\|\Psi x_{n}-\Psi z^{*}\right\|^{2}\right) \\
\leq & \alpha_{n}\left\|f\left(x_{n}\right)-z^{*}\right\|^{2}+\left\|x_{n}-z^{*}\right\|^{2}-\gamma_{n} r_{n}\left(2 \phi-r_{n}\right)\left\|\Psi x_{n}-\Psi z^{*}\right\|^{2} .
\end{aligned}
$$

The same as in (3.30), we have $\left\|\Psi x_{n}-\Psi z^{*}\right\| \rightarrow 0$ as $n \rightarrow \infty$. Likewise, using (3.5), we find

$$
\begin{aligned}
\left\|x_{n+1}-z^{*}\right\|^{2} \leq & \alpha_{n}\left\|f\left(x_{n}\right)-z^{*}\right\|^{2}+\beta_{n}\left\|x_{n}-z^{*}\right\|^{2}+\gamma_{n}\left\|u_{n}-z^{*}\right\|^{2} \\
\leq & \alpha_{n}\left\|f\left(x_{n}\right)-z^{*}\right\|^{2}+\beta_{n}\left\|x_{n}-z^{*}\right\|^{2} \\
& +\gamma_{n}\left(\left\|x_{n}-z^{*}\right\|^{2}-\left\|u_{n}-x_{n}\right\|^{2}+2 r_{n}\left\langle x_{n}-u_{n}, \Psi x_{n}-\Psi z^{*}\right\rangle-r_{n}^{2}\left\|\Psi x_{n}-\Psi z^{*}\right\|^{2}\right) \\
\leq & \alpha_{n}\left\|f\left(x_{n}\right)-z^{*}\right\|^{2}+\left\|x_{n}-z^{*}\right\|^{2}+2 \gamma_{n} r_{n}\left\langle x_{n}-u_{n}, \Psi x_{n}-\Psi z^{*}\right\rangle-\gamma_{n}\left\|u_{n}-x_{n}\right\|^{2} .
\end{aligned}
$$

The same as in (3.34), we have $\lim _{n \rightarrow \infty}\left\|u_{n}-x_{n}\right\|=0$. Since

$$
\begin{aligned}
\left\|x_{n}-S_{n} x_{n}\right\| & \leq\left\|x_{n}-S_{n} y_{n}\right\|+\left\|S_{n} y_{n}-S_{n} u_{n}\right\|+\left\|S_{n} u_{n}-S_{n} x_{n}\right\| \\
& \leq\left\|x_{n}-S_{n} y_{n}\right\|+\left\|y_{n}-u_{n}\right\|+\left\|u_{n}-x_{n}\right\|,
\end{aligned}
$$

we get $\left\|x_{n}-S_{n} x_{n}\right\| \rightarrow 0$ as $n \rightarrow \infty$. From (2.10) and

$$
\begin{aligned}
\left\|S_{n} y_{n}-y_{n}\right\| & \leq\left\|S_{n} y_{n}-S_{n} x_{n}\right\|+\left\|S_{n} x_{n}-x_{n}\right\|+\left\|x_{n}-y_{n}\right\| \\
& \leq\left\|S_{n} x_{n}-x_{n}\right\|+2\left\|x_{n}-y_{n}\right\| \\
& \leq\left\|S_{n} x_{n}-x_{n}\right\|+2\left(\left\|x_{n}-u_{n}\right\|+\left\|u_{n}-y_{n}\right\|\right) \longrightarrow 0,
\end{aligned}
$$

we get $\lim _{n \rightarrow \infty}\left\|S y_{n}-y_{n}\right\|=0$.

Next, we show $\lim \sup _{n \rightarrow \infty}\left\langle f\left(z_{0}\right)-z_{0}, x_{n}-z_{0}\right\rangle \leq 0$, where $z_{0}=P_{\bigcap_{n=1}^{\infty} F\left(T_{n}\right) \cap \Omega \cap V I(A, C)} f\left(z_{0}\right)$. To show this inequality, we choose a subsequence $\left\{y_{n_{i}}\right\}$ of $\left\{y_{n}\right\}$ such that

$$
\limsup _{n \rightarrow \infty}\left\langle f\left(z_{0}\right)-z_{0}, S y_{n}-z_{0}\right\rangle=\lim _{i \rightarrow \infty}\left\langle f\left(z_{0}\right)-z_{0}, S y_{n_{i}}-z_{0}\right\rangle .
$$

Since $\left\{y_{n_{i}}\right\}$ is bounded, there exists a subsequence $\left\{y_{n_{i}}\right\}$ of $\left\{y_{n_{i}}\right\}$ which converges weakly to $z$. Without loss of generality, we can assume that $y_{n_{i}} \rightarrow z$. From $\left\|S y_{n}-y_{n}\right\| \rightarrow 0$, we 
obtain $S y_{n_{i}} \rightarrow z$. We now show that $z \in \bigcap_{n=1}^{\infty} F\left(T_{n}\right) \cap \Omega \cap \operatorname{VI}(A, C)$. Indeed, we observe that $u_{n}=T_{r_{n}}\left(x_{n}-r_{n} \Psi x_{n}\right)$ and

$$
\Phi\left(u_{n}, v\right)+\left\langle\Psi x_{n}, v-u_{n}\right\rangle+\frac{1}{r_{n}}\left\langle v-u_{n}, u_{n}-x_{n}\right\rangle \geq 0 \quad \forall v \in C
$$

From (a2), we deduce that

$$
\left\langle\Psi x_{n}, v-u_{n}\right\rangle+\frac{1}{r_{n}}\left\langle v-u_{n}, u_{n}-x_{n}\right\rangle \geq-\Phi\left(u_{n}, v\right)=\Phi\left(v, u_{n}\right)
$$

and hence

$$
\left\langle\Psi x_{n_{i}}, v-u_{n_{i}}\right\rangle+\left\langle v-u_{n_{i}}, \frac{u_{n_{i}}-x_{n_{i}}}{r_{n_{i}}}\right\rangle \geq \Phi\left(v, u_{n_{i}}\right) .
$$

Form $\lim _{n \rightarrow \infty}\left\|u_{n}-y_{n}\right\|=0$, we get $u_{n_{i}} \rightarrow z$. Put $z_{t}=t v+(1-t) z$ for all $t \in(0,1]$ and $v \in C$. Consequently, we get $z_{t} \in C$. From (3.42), it follows that

$$
\begin{aligned}
\left\langle\Psi z_{t}, z_{t}-u_{n_{i}}\right\rangle \geq & \left\langle\Psi z_{t}, z_{t}-u_{n_{i}}\right\rangle-\left\langle\Psi x_{n_{i}}, z_{t}-u_{n_{i}}\right\rangle-\left\langle z_{t}-u_{n_{i}}, \frac{u_{n_{i}}-x_{n_{i}}}{r_{n_{i}}}\right\rangle+\Phi\left(z_{t}, u_{n_{i}}\right) \\
= & \left\langle\Psi z_{t}-\Psi u_{n_{i}}, z_{t}-u_{n_{i}}\right\rangle+\left\langle\Psi u_{n_{i}}-\Psi x_{n_{i}}, z_{t}-u_{n_{i}}\right\rangle \\
& -\left\langle z_{t}-u_{n_{i}}, \frac{u_{n_{i}}-x_{n_{i}}}{r_{n_{i}}}\right\rangle+\Phi\left(z_{t}, u_{n_{i}}\right) .
\end{aligned}
$$

From the Lipschitz continuous of $\Psi$ and $\lim _{n \rightarrow \infty}\left\|u_{n}-x_{n}\right\|=0$, we obtain $\left\|\Psi u_{n_{i}}-\Psi x_{n_{i}}\right\| \rightarrow 0$. Since $\Psi$ is monotone, we know that $\left\langle\Psi z_{t}-\Psi u_{n_{i}}, z_{t}-u_{n_{i}}\right\rangle \geq 0$. Further, $\left(u_{n_{i}}-x_{n_{i}}\right) / r_{n_{i}} \rightarrow 0$. It follows from (a4) that

$$
\Phi\left(z_{t}, z\right) \leq \lim _{i \rightarrow \infty} \Phi\left(z_{t}, u_{n_{i}}\right) \leq \lim _{i \rightarrow \infty}\left\langle\Psi z_{t}, z_{t}-u_{n_{i}}\right\rangle=\left\langle\Psi z_{t}, z_{t}-z\right\rangle
$$

Owing to (a1) and (a4), we get that

$$
\begin{aligned}
0 & =\Phi\left(z_{t}, z_{t}\right) \leq t \Phi\left(z_{t}, v\right)+(1-t) \Phi\left(z_{t}, z\right) \\
& \leq t \Phi\left(z_{t}, v\right)+(1-t)\left\langle\Psi z_{t}, z_{t}-z\right\rangle \\
& \leq t \Phi\left(z_{t}, v\right)+(1-t) t\left\langle\Psi z_{t}, v-z\right\rangle
\end{aligned}
$$

and hence

$$
\Phi\left(z_{t}, v\right)+(1-t)\left\langle\Psi z_{t}, v-z\right\rangle \geq 0 .
$$


Letting $t \rightarrow 0$, we have

$$
\Phi(z, v)+\langle\Psi z, v-z\rangle \geq 0
$$

This implies that $z \in \Omega$.

Furthermore, we prove that $z \in F(S)=\bigcap_{n=1}^{\infty} F\left(T_{n}\right)$. Assume $z \neq S z$, since $\lim _{n \rightarrow \infty} \| u_{n}-$ $x_{n} \|=0$, we have $x_{n_{i}} z$. From Opial's theorem [27], we get

$$
\begin{aligned}
\liminf _{i \rightarrow \infty}\left\|x_{n_{i}}-z\right\| & <\liminf _{i \rightarrow \infty}\left\|x_{n_{i}}-S z\right\| \\
& \leq \liminf _{i \rightarrow \infty}\left(\left\|x_{n_{i}}-S x_{n_{i}}\right\|+\left\|S x_{n_{i}}-S z\right\|\right) \\
& \leq \liminf _{i \rightarrow \infty}\left\|x_{n_{i}}-z\right\| .
\end{aligned}
$$

This is a contradiction. Hence, $z \in F(S)=\bigcap_{n=1}^{\infty} F\left(T_{n}\right)$.

Now, we will show that $z \in \operatorname{VI}(A, C)$. Let

$$
T u= \begin{cases}A u+N_{C} u, & u \in C, \\ \emptyset, & u \text { is not in } C .\end{cases}
$$

Then $T$ is a maximal monotone [23]. Let $(u, w) \in G(T)$, since $w-A u \in N_{C}(u)$ and $y_{n} \in C$, we have $\left\langle u-y_{n}, w-A u\right\rangle \geq 0$. From $y_{n}=P_{C}\left(u_{n}-\lambda_{n} A u_{n}\right)$, we have

$$
\left\langle u-y_{n}, y_{n}-\left(u_{n}-\lambda_{n} A u_{n}\right)\right\rangle \geq 0
$$

This is,

$$
\left\langle u-y_{n}, \frac{y_{n}-u_{n}}{\lambda_{n}}+A u_{n}\right\rangle \geq 0
$$

Therefore, we obtain

$$
\begin{aligned}
\left\langle u-y_{n_{i}}, w\right\rangle & \geq\left\langle u-y_{n_{i}}, A u\right\rangle \\
& \geq\left\langle u-y_{n_{i}}, A u\right\rangle-\left\langle u-y_{n_{i}}, \frac{y_{n_{i}}-u_{n_{i}}}{\lambda_{n_{i}}}+A u_{n_{i}}\right\rangle \\
& =\left\langle u-y_{n_{i}}, A u-A u_{n_{i}}-\frac{y_{n_{i}}-u_{n_{i}}}{\lambda_{n_{i}}}\right\rangle \\
& =\left\langle u-y_{n_{i}}, A u-A y_{n_{i}}\right\rangle+\left\langle u-y_{n_{i}}, A y_{n_{i}}-A u_{n_{i}}\right\rangle-\left\langle u-y_{n_{i}}, \frac{y_{n_{i}}-u_{n_{i}}}{\lambda_{n_{i}}}\right\rangle \\
& \geq\left\langle u-y_{n_{i}}, A y_{n_{i}}-A u_{n_{i}}\right\rangle-\left\langle u-y_{n_{i}}, \frac{y_{n_{i}}-u_{n_{i}}}{\lambda_{n_{i}}}\right\rangle .
\end{aligned}
$$


Fixed Point Theory and Applications

Noting that $\lim _{n \rightarrow \infty}\left\|u_{n}-y_{n}\right\|=0$ and $A$ is Lipschitz continuous, we obtain

$$
\langle u-z, w\rangle \geq 0
$$

Since $T$ is maximal monotone, we have $z \in T^{-1} 0$ and so $z \in \operatorname{VI}(A, C)$. Thus, $z \in \bigcap_{n=1}^{\infty} F\left(T_{n}\right) \cap$ $\Omega \cap \operatorname{VI}(A, C)$. The property of the metric projection implies that

$$
\begin{aligned}
\limsup _{n \rightarrow \infty}\left\langle f\left(z_{0}\right)-z_{0}, x_{n}-z_{0}\right\rangle & =\limsup _{n \rightarrow \infty}\left\langle f\left(z_{0}\right)-z_{0}, S_{n} y_{n}-z_{0}\right\rangle \\
& =\limsup _{n \rightarrow \infty}\left\langle f\left(z_{0}\right)-z_{0}, S y_{n}-z_{0}\right\rangle \\
& =\lim _{i \rightarrow \infty}\left\langle f\left(z_{0}\right)-z_{0}, S y_{n_{i}}-z_{0}\right\rangle \\
& =\left\langle f\left(z_{0}\right)-z_{0}, z-z_{0}\right\rangle \leq 0 .
\end{aligned}
$$

From (3.1) we obtain

$$
\begin{aligned}
\left\|x_{n+1}-z_{0}\right\|^{2}= & \left\langle\alpha_{n} f\left(x_{n}\right)+\beta_{n} x_{n}+\gamma_{n} S_{n} y_{n}-z_{0}, x_{n+1}-z_{0}\right\rangle \\
= & \alpha_{n}\left\langle f\left(x_{n}\right)-z_{0}, x_{n+1}-z_{0}\right\rangle+\beta_{n}\left\langle x_{n}-z_{0}, x_{n+1}-z_{0}\right\rangle+\gamma_{n}\left\langle S_{n} y_{n}-z_{0}, x_{n+1}-z_{0}\right\rangle \\
\leq & \alpha_{n}\left\langle f\left(x_{n}\right)-f\left(z_{0}\right), x_{n+1}-z_{0}\right\rangle+\alpha_{n}\left\langle f\left(z_{0}\right)-z_{0}, x_{n+1}-z_{0}\right\rangle \\
& +\frac{1}{2} \beta_{n}\left(\left\|x_{n}-z_{0}\right\|^{2}+\left\|x_{n+1}-z_{0}\right\|^{2}\right)+\frac{1}{2} \gamma_{n}\left(\left\|S_{n} y_{n}-z_{0}\right\|^{2}+\left\|x_{n+1}-z_{0}\right\|^{2}\right) \\
\leq & \frac{1}{2}\left(1-\alpha_{n}\right)\left(\left\|x_{n}-z_{0}\right\|^{2}+\left\|x_{n+1}-z_{0}\right\|^{2}\right)+\alpha_{n}\left\langle f\left(z_{0}\right)-z_{0}, x_{n+1}-z_{0}\right\rangle \\
& +\frac{1}{2} \alpha_{n}\left(\left\|f\left(x_{n}\right)-f\left(z_{0}\right)\right\|^{2}+\left\|x_{n+1}-z_{0}\right\|^{2}\right) \\
\leq & \frac{1}{2}\left(1-\alpha_{n}\left(1-\alpha^{2}\right)\right)\left\|x_{n}-z_{0}\right\|^{2}+\frac{1}{2}\left\|x_{n+1}-z_{0}\right\|^{2}+\alpha_{n}\left\langle f\left(z_{0}\right)-z_{0}, x_{n+1}-z_{0}\right\rangle
\end{aligned}
$$

which implies that

$$
\left\|x_{n+1}-z_{0}\right\|^{2} \leq\left(1-\alpha_{n}\left(1-\alpha^{2}\right)\right)\left\|x_{n}-z_{0}\right\|^{2}+2 \alpha_{n}\left\langle f\left(z_{0}\right)-z_{0}, x_{n+1}-z_{0}\right\rangle .
$$

Setting

$$
\delta_{n}=2 \alpha_{n}\left\langle f\left(z_{0}\right)-z_{0}, x_{n+1}-z_{0}\right\rangle,
$$

we have

$$
\limsup _{n \rightarrow \infty} \frac{\delta_{n}}{\alpha_{n}\left(1-\alpha^{2}\right)} \leq 0
$$


Applying Lemma 2.8 to (3.56), we conclude that $\left\{x_{n}\right\}$ converges strongly to $z_{0}$. Consequently, $\left\{u_{n}\right\}$ and $\left\{y_{n}\right\}$ converge strongly to $z_{0}$. This completes the proof.

As direct consequences of Theorem 3.1, we have the following two corollaries.

Corollary 3.2. Let $C$ be a nonempty closed convex subset of a real Hilbert space $H$. Let $f: C \rightarrow C$ be a contraction mapping with Lipschitz constant $\alpha \in[0,1)$, and let $A: C \rightarrow H$ be an inverse-strongly monotone mapping with constant $\rho>0$. Suppose $x_{1} \in C$ and $\left\{x_{n}\right\}$ generated by

$$
x_{n+1}=\alpha_{n} f\left(x_{n}\right)+\beta_{n} x_{n}+\gamma_{n} P_{C}\left(x_{n}-\lambda_{n} A x_{n}\right)
$$

for all $n \in \mathbb{N}$, where $\left\{\alpha_{n}\right\},\left\{\beta_{n}\right\},\left\{\gamma_{n}\right\}$ are three sequences in $[0,1],\left\{\lambda_{n}\right\}$ is a sequence in $(0, b]$ for some $0<b<2 \rho$ satisfying conditions (i)-(iv). Then $\left\{x_{n}\right\}$ converges strongly to the point $x^{*} \in \operatorname{VI}(A, C)$, where $x^{*}=P_{V I(A, C)} f\left(x^{*}\right)$.

Proof. Let $\Phi(u, v)=0$ and $\Psi x=0$ for all $u, v, x \in C$ and $r_{n}=1$ in Theorem 3.1. Then $u_{n}=x_{n}$ for $n=1,2, \ldots$ Letting $T_{n}=I$ (the identity mapping) for all $n \in \mathbb{N}$, then $S_{n}=I$ for $n=1,2, \ldots$ It is easy to see that all conditions of Theorem 3.1 hold. Therefore, we know that the sequence $\left\{x_{n}\right\}$ generated by (3.59) converges strongly to $x^{*}=P_{\mathrm{VI}(A, C)} f\left(x^{*}\right)$. This completes the proof.

Remark 3.3. From Corollary 3.2, we can get an iterative scheme for finding the solution of the variational inequality involving the $\alpha$-inverse-strongly monotone mapping $A$.

Corollary 3.4 (see [17, Theorem 3.5]). Let $C$ be a nonempty closed convex subset of a real Hilbert space $H$. Let $\Phi$ be a bifunction from $C \times C$ into $\mathbb{R}$ satisfying (a1)-(a4), $f: C \rightarrow C$ a contraction mapping with constant $\alpha \in[0,1)$. Let $S_{n}: C \rightarrow C$ be an $S$-mapping generated by $T_{1}, T_{2}, \ldots$ and $\pi_{1}, \pi_{2}, \ldots$ and $\bigcap_{n=1}^{\infty} F\left(T_{n}\right) \cap E P(\Phi) \neq \emptyset$, where sequence $\left\{T_{n}\right\}$ is nonexpansive and $\left\{\pi_{n}\right\}$ is a sequence in $(0, \sigma]$ for some $\sigma \in(0,1)$. Suppose $x_{1} \in C$ and $\left\{x_{n}\right\},\left\{u_{n}\right\}$ are generated by

$$
\begin{gathered}
\Phi\left(u_{n}, v\right)+\frac{1}{r_{n}}\left\langle v-u_{n}, u_{n}-x_{n}\right\rangle \geq 0 \quad \forall v \in C, \\
x_{n+1}=\alpha_{n} f\left(x_{n}\right)+\beta_{n} x_{n}+\gamma_{n} S_{n} u_{n}
\end{gathered}
$$

for all $n \in \mathbb{N}$, where $\left\{\alpha_{n}\right\},\left\{\beta_{n}\right\},\left\{\gamma_{n}\right\}$ are three sequences in $[0,1]$, and $\left\{r_{n}\right\}$ is a sequence in $(0,+\infty)$ satisfying conditions (i)-(iii) and (v). Then, the sequences $\left\{x_{n}\right\}$ and $\left\{u_{n}\right\}$ converge strongly to the point $x^{*} \in \bigcap_{n=1}^{\infty} F\left(T_{n}\right) \cap E P(\Phi)$, where $x^{*}=P_{\bigcap_{n=1}^{\infty} F\left(T_{n}\right) \cap E P(\Phi)} f\left(x^{*}\right)$.

Proof. Let $\lambda_{n}=1$ for $n=1,2, \ldots$ and $\Psi(x)=0$ and $A(x)=0$ for all $x \in C$ in Theorem 3.1. Since $u_{n} \in C$, we get that $u_{n}=P_{C} u_{n}$. It follows from Theorem 3.1 that the sequences $\left\{x_{n}\right\}$ and $\left\{u_{n}\right\}$ converge strongly to the point $x^{*}=P_{\bigcap_{n=1}^{\infty} F\left(T_{n}\right) \cap \mathrm{EP}(\Phi)} f\left(x^{*}\right)$. This completes the proof.

Remark 3.5. The main result of Yao et al. [17, Corollary 3.2] improved and extended the corresponding theorems in Combettes and Hirstoaga [3] and S. Takahashi and W. Takahashi [14]. 
Our Theorem 3.1 improves and extends Theorem 3.5 of Yao et al. [17] in the following aspects:

(1) the equilibrium problem is extended to the generalized equilibrium problem;

(2) our iterative process (3.1) is different from Yao et al. iterative process (3.60) because there are a project operator and an $\alpha$-inverse-strongly monotone mapping;

(3) our iterative process (3.1) is more general than Yao et al. iterative process (3.60) because it can be applied to solving the problem of finding a common element of the set of solutions of generalized equilibrium problems, the set of common fixed points of infinitely many nonexpansive mappings, and the set of solutions of the variational inequality for $\alpha$-inverse-strongly monotone mapping.

\section{Acknowledgments}

This work was supported by the National Natural Science Foundation of China (50674078, 50874096, 10671135, 70831005) and the Open Fund of State Key Laboratory of Oil and Gas Reservoir Geology and Exploitation (Southwest Petroleum University).

\section{References}

[1] E. Blum and W. Oettli, "From optimization and variational inequalities to equilibrium problems," The Mathematics Student, vol. 63, no. 1-4, pp. 123-145, 1994.

[2] J. Li, N.-J. Huang, and J. K. Kim, “On implicit vector equilibrium problems," Journal of Mathematical Analysis and Applications, vol. 283, no. 2, pp. 501-512, 2003.

[3] P. L. Combettes and S. A. Hirstoaga, "Equilibrium programming in Hilbert spaces," Journal of Nonlinear and Convex Analysis, vol. 6, no. 1, pp. 117-136, 2005.

[4] S. D. Flåm and A. S. Antipin, "Equilibrium programming using proximal-like algorithms," Mathematical Programming, vol. 78, no. 1, pp. 29-41, 1997.

[5] K.-Q. Wu, N.-J. Huang, and J.-C. Yao, "Existence theorems of solutions for a system of nonlinear inclusions with an application," Journal of Inequalities and Applications, vol. 2007, Article ID 56161, 12 pages, 2007.

[6] L. C. Zeng, S. Schaible, and J. C. Yao, "Iterative algorithm for generalized set-valued strongly nonlinear mixed variational-like inequalities," Journal of Optimization Theory and Applications, vol. 124, no. 3, pp. 725-738, 2005.

[7] F. E. Browder and W. V. Petryshyn, "Construction of fixed points of nonlinear mappings in Hilbert space," Journal of Mathematical Analysis and Applications, vol. 20, pp. 197-228, 1967.

[8] W. Takahashi, Nonlinear Functional Analysis, Fixed Point Theory and Its Applications, Yokohama, Yokohama, Japan, 2000.

[9] S. D. Flåm and A. S. Antipin, "Equilibrium programming using proximal-like algorithms," Mathematical Programming, vol. 78, no. 1, pp. 29-41, 1997.

[10] A. N. Iusem and W. Sosa, "Iterative algorithms for equilibrium problems," Optimization, vol. 52, no. 3, pp. 301-316, 2003.

[11] N.-J. Huang, H.-Y. Lan, and K. L. Teo, “On the existence and convergence of approximate solutions for equilibrium problems in Banach spaces," Journal of Inequalities and Applications, vol. 2007, Article ID 17294, 14 pages, 2007.

[12] W. Takahashi and M. Toyoda, "Weak convergence theorems for nonexpansive mappings and monotone mappings," Journal of Optimization Theory and Applications, vol. 118, no. 2, pp. 417-428, 2003.

[13] H. Iiduka and W. Takahashi, "Strong convergence theorems for nonexpansive mappings and inversestrongly monotone mappings," Nonlinear Analysis: Theory, Methods E Applications, vol. 61, no. 3, pp. 341-350, 2005. 
[14] S. Takahashi and W. Takahashi, "Viscosity approximation methods for equilibrium problems and fixed point problems in Hilbert spaces," Journal of Mathematical Analysis and Applications, vol. 331, no. 1, pp. 506-515, 2007.

[15] R. Wittmann, "Approximation of fixed points of nonexpansive mappings," Archiv der Mathematik, vol. 58, no. 5, pp. 486-491, 1992.

[16] A. Tada and W. Takahashi, "Weak and strong convergence theorems for a nonexpansive mapping and an equilibrium problem," Journal of Optimization Theory and Applications, vol. 133, no. 3, pp. 359-370, 2007.

[17] Y. Yao, Y.-C. Liou, and J.-C. Yao, “Convergence theorem for equilibrium problems and fixed point problems of infinite family of nonexpansive mappings," Fixed Point Theory and Applications, vol. 2007, Article ID 64363, 12 pages, 2007.

[18] A. Moudafi, "Weak convergence theorems for nonexpansive mappings and equilibrium problems," Journal of Nonlinear and Convex Analysis, vol. 9, no. 1, pp. 37-43, 2008.

[19] J.-W. Peng and J.-C. Yao, "A new hybrid-extragradient method for generalized mixed equilibrium problems, fixed point problems and variational inequality problems," Taiwanese Journal of Mathematics, vol. 12, no. 6, pp. 1401-1432, 2008.

[20] J.-W. Peng, Y. Wang, D. S. Shyu, and J.-C. Yao, "Common solutions of an iterative scheme for variational inclusions, equilibrium problems, and fixed point problems," Journal of Inequalities and Applications, vol. 2008, Article ID 720371, 15 pages, 2008.

[21] S. Takahashi and W. Takahashi, "Strong convergence theorem for a generalized equilibrium problem and a nonexpansive mapping in a Hilbert space," Nonlinear Analysis: Theory, Methods E Applications, vol. 69, no. 3, pp. 1025-1033, 2008.

[22] W.-Y. Zeng, N.-J. Huang, and C.-W. Zhao, "Viscosity approximation methods for generalized mixed equilibrium problems and fixed points of a sequence of nonexpansive mappings," Fixed Point Theory and Applications, vol. 2008, Article ID 714939, 15 pages, 2008.

[23] R. T. Rockafellar, "On the maximality of sums of nonlinear monotone operators," Transactions of the American Mathematical Society, vol. 149, pp. 75-88, 1970.

[24] K. Shimoji and W. Takahashi, "Strong convergence to common fixed points of infinite nonexpansive mappings and applications," Taiwanese Journal of Mathematics, vol. 5, no. 2, pp. 387-404, 2001.

[25] T. Suzuki, "Strong convergence theorems for infinite families of nonexpansive mappings in general Banach spaces," Fixed Point Theory and Applications, vol. 2005, no. 1, pp. 103-123, 2005.

[26] H.-K. Xu, "Viscosity approximation methods for nonexpansive mappings," Journal of Mathematical Analysis and Applications, vol. 298, no. 1, pp. 279-291, 2004.

[27] Z. Opial, "Weak convergence of the sequence of successive approximations for nonexpansive mappings," Bulletin of the American Mathematical Society, vol. 73, pp. 591-597, 1967. 